\title{
Optimal Allocation and Sizing of Distributed Generation in Radial Distribution Systems using Geolocation-Aware Heuristic Optimization
}

\author{
Ali M. Al-Jumaili \\ Department of Electrical and \\ Electronic Engineering Dumlupınar \\ University
}

\author{
Yilmaz Aslan \\ Department of Electrical and \\ Electronic Engineering \\ Dumlupınar University
}

\author{
Celal Yaşar \\ Department of Electrical and \\ Electronic Engineering \\ Dumlupınar University
}

\begin{abstract}
With the growing need of energy and the recent concerns about global warming, attention has been brought towards the integration of renewable energy sources into existing power grids. The optimal location of the units and their sizes are significant parameters that influence the overall performance of the power grid. Most of the recent placement and sizing methods rely on using heuristic optimization algorithms, which rely on particles that are randomly deployed in the search space in order to recognize the optimal values that minimizes the cost value. In this study, a geolocation-aware representation of the power grid is proposed, which allows the optimizers to conduct a more efficient search, as the changes of the values matches the movement of the particles in the search space. Hence, the proposed approach has been able to significantly improve the performance of the optimizers, which in return has significantly improved the characteristics of the power grid. Accordingly, the proposed approach has been able to improve the IEEE 33 bus radial distribution test system by reducing the loss to $6.7 \mathrm{KW}$, compared to the original 24.97, by adding two generation units, with significant improvement in the voltage profile, with minimum voltage of $0.9976 \mathrm{Pu}$ and maximum and average voltage of $1 \mathrm{Pu}$.
\end{abstract}

\section{Keywords}

Distributed generation, Radial distribution system,Optimal size, Optimal location, Active power losses.

\section{INTRODUCTION}

With the dramatic influence of using large centralized generation units that rely on fossil fuel to energize power grids [1-3], significant attention has been brought towards the use of renewable energy to satisfy the growing demand on energy $[4,5]$. Generation units that rely on renewable energy are normally of smaller sizes and are distributed around the grid, which has led to the paradigm of Distributed Generation (DG), which is the main mean of integrating these resources into the distribution system. Instead of generating the power in remote areas and transmitting the power to the consumers, these resources are located closer to the consumers to improve the quality of the power being provided over the power grid $[6,7]$. The active and reactive power that are injected by the DG units to the grid have several benefits to the grid, such as improving reliability and security, as well as reducing system oscillations. However, the main two benefits that DG has provided to existing centralized power grids is reducing the losses, caused by the need to transmit the power from the remote centralized units to the consumers, and improve the voltage profile. The significance of these features relies mainly on the positioning of the DG resource being connected to the grid. Accordingly, several researches have been conducted to investigate different methodologies that can optimize the placement of these resources [8].Heuristic optimization algorithms are widely used to optimize the placement of these resources. These algorithms do not interact with the operation of the system they are interacting with, instead, they only adjust the parameters that affect the operation of the system and monitor its performance, without the need to recognize how these parameters affect the operation of the system. The main aim of these algorithms is to balance the exploration of the search space and its exploitations, so that, the optimal values of the system parameters are recognized without the need to search for all possible combinations. Most of these algorithms, such as the Particle Swarm Optimizer (PSO) and Artificial Bee Colony $(\mathrm{ABC})$, are inspired by the way these swarms conduct searches for food. Other algorithms, such as the Sine-Cosine Algorithm (SCA), use specially designed mathematical models that attempt to balance the exploration and exploitation. However, all of these methods rely on finding the position of the parameter values that produce the best fitness, locally and globally, and rely on these values in the search that is conducted in the next iteration [8-10].

\subsection{Problem Formation}

Despite the ability of several of the recent methods to optimize the placement of DG resources, in order to reduce losses and improve the voltage profile of the grid, the reliance on the busbar number in the optimization limits the ability of the optimization algorithms to conduct an efficient search, i.e., achieve balanced exploration and exploitation. In addition to the higher complexity and computer resources that are required by these optimization algorithms to recognize the optimal location of the DG resource, there is a significant chance of missing the actual optimal position in complex reallife scenarios. For instance, in the IEEE 69-bus distribution test system, shown in Figure 1, the search space for optimal position of the DG resource to reduce losses is shown in Figure 2. As figure 2 shows, a particle moving from busbar 10 to busbar 20 can continue searching in that direction, as the cost value, i.e., total loss, is being reduced. However, a particle that is moving from busbar 20 toward busbar 30 may terminate the search in that direction and change its direction after noticing a significant increment in the cost value. Alternatively, the hypothesis of this study is that presenting the search space of the DG grid to the optimization algorithm based on the geolocation of the busbars, as shown in Figure 3, a significant improvement can be achieved in the operation of the optimization procedure, which allows recognizing the 
optimal location faster, without missing the actual optimal

position, even in complex grids.

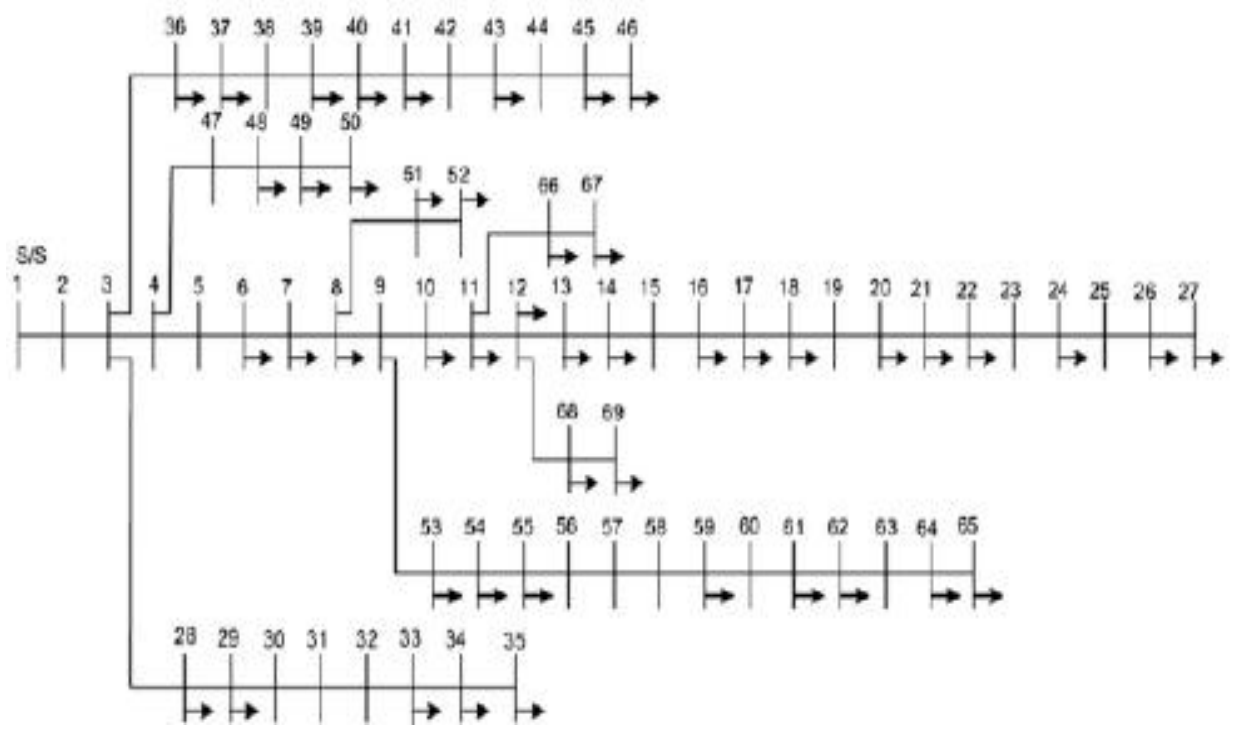

Fig1: The IEEE 69-bus distribution test system [11].

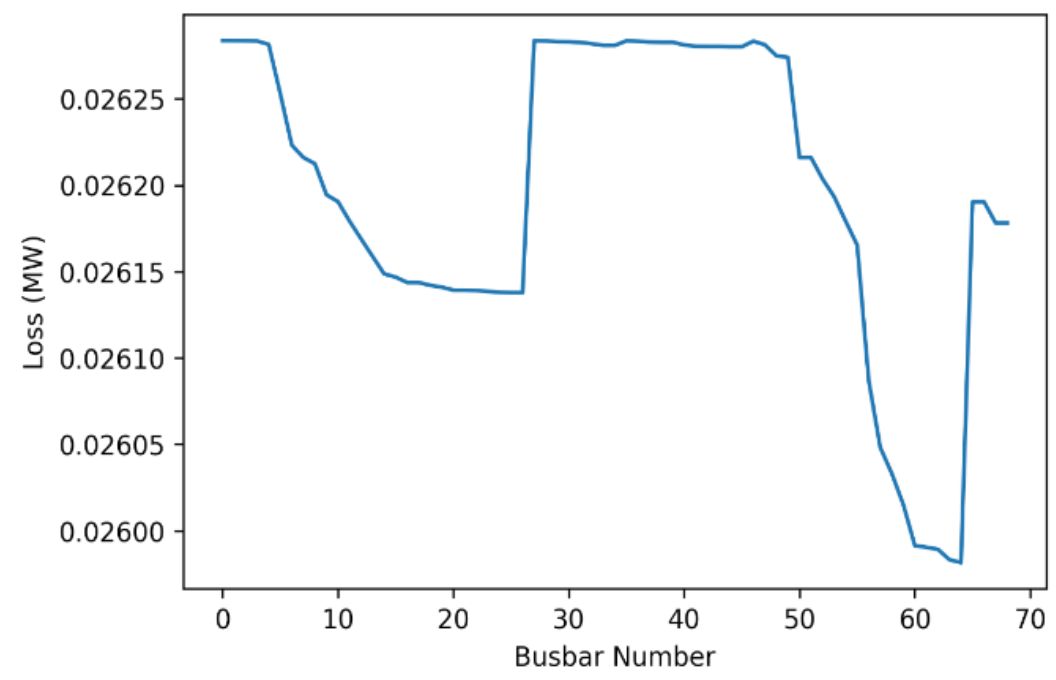

Fig2: Total loss versus busbar number in which DG is positioned.

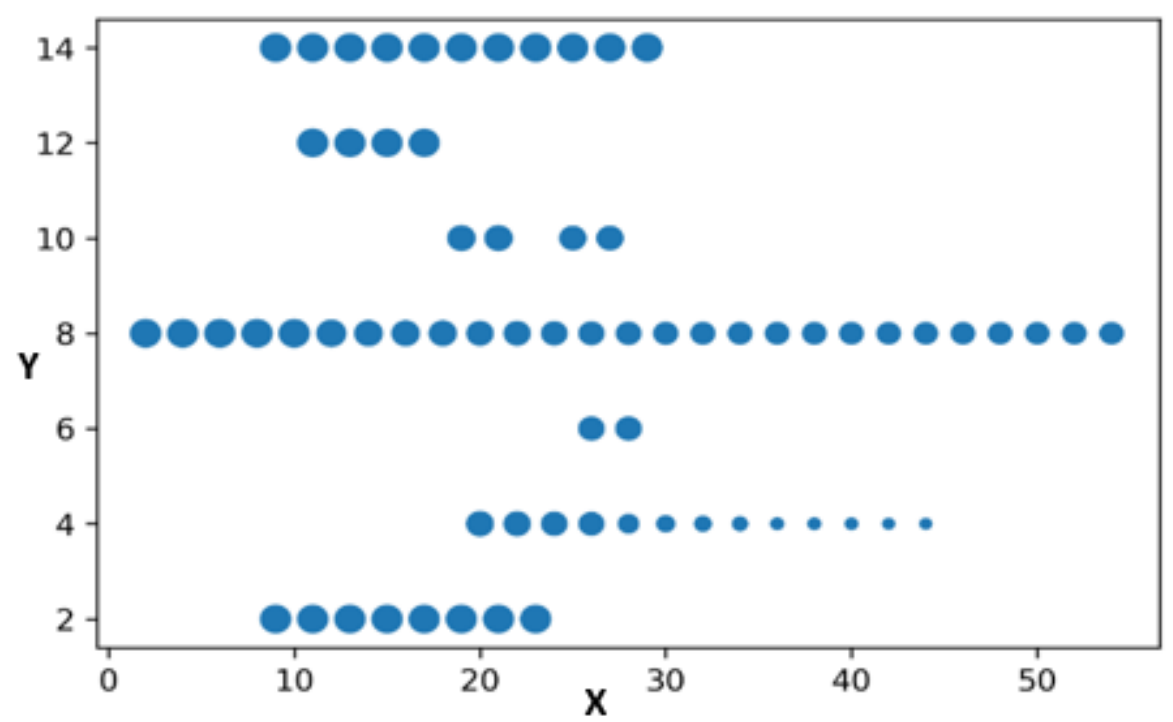

Fig3: Geolocation-based search space for the IEEE 69-bus distribution test system 


\section{MATERIALS}

In order to validate the hypothesis of this study, three optimization functions are selected to compare their performance using the standard approach, in which the busbar number is used, and when the geolocation of the busbar is used during optimization.

\subsection{Particle Swarm Optimization (PSO)}

Particle Swarm Optimization (PSO) is one of the widely used algorithms to solve optimization problems, where a search space is generated based on the limits of the possible values for the parameters of the system. Then, a search is conducted in that space for the parameters values that produce the optimal performance of the system. To search for these values, a predefined number of particles are distributed randomly in that search space and the performance of the system is measured per each particle. For this initial step, the information per each particle is stored as the local best, which indicate that these parameters values have achieved the best system performance per this particle, in addition to the global best performance, where the information of the particle that has achieved the best system performance is stored in it [12, 13].

Next, the PSO algorithm iterates through a predefined number of iterations, where per each iteration the position of each particle is adjusted and the system performance at that position is measured. If the system has a better performance at the new position, then the local best of the particle is updated using the information at the new position. By the end of the iteration, the best system performance achieved by any particle in the swarm is compared to the global best, where the information of the global best is updated using that particle's information, if it has achieved better performance [14].

To ensure balanced exploration and exploitation of the search space by the swarm particle, the position of each particle is updated per iteration using three factors, which are the last velocity of the particle in the previous iteration, the position of the local best of the particle, and the position of the global best achieved by the particles so far, as well as two random number to ensure the exploration. Moreover, three more parameters are considered, in the equation the calculates the velocity of the particle, to allow more control on the positioning of the particle in the search space. The first parameter controls the effect of the latest velocity of the particle, i.e. in the previous iteration, which is known as the inertia. For better exploitation, the value of the inertia $(w)$ is decreased, within a certain range, as more iterations are completed by the algorithm. Moreover, the parameters $c_{l}$ and $c_{2}$ control the effect of the distance between the current position and the local and global bests, respectively [15]. Equation (1) shows the computations required to update the velocity $(V)$ of the $\mathrm{i}^{\text {th }}$ particles in the search space, where $P$ is the local best of that particle, $G$ is the global best, $X$ is the position of the particle at iteration $t, G$ is the position of the global best, while $r_{l}$ and $r_{2}$ are two random numbers that are generated per each particle per iteration. This velocity is used to calculate the new position of that particle, using equation (2) [15].

$$
\begin{gathered}
\overrightarrow{V_{l}^{t+1}}=w \overrightarrow{V_{l}^{t}}+c_{1} r_{1}\left(\overrightarrow{P_{l}^{t}}-\overrightarrow{X_{l}^{t}}\right)+c_{2} r_{2}\left(\overrightarrow{G^{t}}-\overrightarrow{X_{l}^{t}}\right) \\
\overrightarrow{X_{l}^{t+1}}=\overrightarrow{X_{l}^{t}}+\overrightarrow{V_{l}^{t+1}}
\end{gathered}
$$

Algorithm 1 illustrates the main steps executed by the PSO in order to find the optimal parameters that optimize the performance of a system.

Algorithm 1: The Particle Swarm Optimization algorithm.

Begin
Step 1: Generate a search space according to the limits of
the parameters.
Step 2: Generate $M$ random particles $P$ in the search
space, with dimension $D$, each.
Step 3: For each particle in $M$
Calculate the fitness of the particle $F_{m}^{0}$.
$\quad$ Update local best information $p b e s t_{m} \leftarrow F_{m}^{0}$.
Step 4: Select particle information with the best fitness as
the global best $G$.
Step 5: While the maximum number of iterations $T$ is not
reached
For each particle
Calculate velocity $v_{m}^{t}$ using Eq. 2.7 .
Calculate new position $X_{m}^{t}$ using Eq. 2.8 .
Measure the fitness of the particle.
If the new fitness $F_{m}^{t}$ is better than the local best
fitness
Update local best $p b e s t_{m} \leftarrow F_{m}^{t}$
If the new fitness $F_{m}^{t}$ is better than the global
best fitness $G \leftarrow P_{m}^{t}$
Step

\subsection{Artificial Bee Colony}

The colony of the artificial bees in the Artificial Bee Colony $(\mathrm{ABC})$ algorithm consists of three groups of bees, which are the employed, onlookers and scout bees. Onlooker bees wait on the dance area to make decisions about the food sources selection while employed bees are the ones that go to food sources that they have already visited earlier. Scout bees are responsible of carrying out random searches for food sources in the defined space. Initially, half of the bees in the colony are set as employed bees while the other half is set as onlookers. The employed bees are distributed on the food sources around the hive, where each bee is assigned to a single source, where a bee assigned to a food source that becomes exhausted becomes a scout. As soon as a bee becomes a scout, it starts searching for new food sources [16].

Per each cycle, the employed bee is sent to the food sources to measure the nectar amount of that source. Then, the onlooker bees select the food sources after collecting the information collected by the employed workers. Additionally, scout bees are recognized and sent to possible food sources to measure their nectar amounts. Initially, when no information is available about the space around the hive, scout bees are sent to random locations to measure their nectar amounts and come back to the hive. Based on the measured amounts, the onlooker bees make the decision and send the employed bees to the sources they have found previously that persist in their memories and investigate for better food sources, visually. Finding a food source with higher amount of nectar increases 
the probability of selecting that source by the onlooker bees. If that food source is selected, the employed bee is directed to that source.

The food source position within the ABC algorithm symbolizes a potential solution to optimize the problem, while there is a corresponding nectar quantity of the food source (fitness) regarding the related solution. The amount of onlooker bees or the employed bees remain equal to the amount of population solutions. A randomly distributed first population was generated by $\mathrm{ABC}$ in the first step $P(G=0)$ of SN solutions (the positions of the food source), in which $\mathrm{SN}$ stands for the population size. For every solution the $D$ dimensional vector is each solution (food source) $x i(i=1,2$, . $\ldots, \mathrm{SN}$ ), in which the D represents the amount of optimization parameters. When initialization is complete, there is a subjection of the position population to the repeated cycles, $C$ $=1,2, \ldots, C$ max, regarding the search procedures of the adopted bees, scout bees, and the onlooker bees. A modification regarding the position is generated by the onlooker be or the artificial employed bee in a probabilistic manner in her memory to seek for the new source of food and get the nectar amount tested, which is the fitness value regarding the new source. Considering real bees, new food sources are produced on the basis of the comparison process regarding the food source in a specific territory based on the information the bee puts together, visually.

Our model involves the generation of the position regarding a new food source on the basis of the comparison process regarding positions of food sources. Nevertheless, the model also shows no implementation of any information by the artificial bees for comparison. The source position of the food is selected randomly and a modification is generated on the existing one in their memory as provided in (2.10). As long as there is a higher nectar quantity of the new source compared to the former one memorized by the bee while forgetting the previous one. If not, the previous position will be kept.

Once the search process has been completed by all the employed bees, the nectar information regarding the sources of food is shared by them and the information of their positions with the onlooker bees are set for the dance spot. The nectar information obtained from every employed bees is assessed by the onlooker bee ad a food source is selected with the probability associated to the amount of its nectar. Just like the employed bee, a modification is generated regarding the position in her memory and the amount of the nectar is checked regarding the source of candidate. Based on the fact that there is a higher center compared to the previous one, the new position is memorized by the bee while the old one is forgotten. A food source is selected by the onlooker bee based on the probability value related to the food source, a calculation of pi is done as presented in Equation (3) [16].

$$
p_{i}=\frac{f i t_{i}}{\sum_{n=1}^{S N} f i t_{n}}
$$

In which the fit $i$ is considered the solution $i$ fitness value assessed by the employed bee, typically proportional to the food source nectar amount within the position $I$ while the amount of food sources remains SN, typically the same as the amount of employed bees (BN). The employed bees in this manner get their information exchanged with the onlookers. For the candidate food position to be generated from the previous one, Equation 4) is used by $\mathrm{ABC}$ [16].

$$
v_{i j}=x_{i j}+\emptyset_{i j}\left(x_{i j}-x_{k j}\right)
$$

Where $k \in\{1,2, \ldots, \mathrm{BN}\}$ and $j \in\{1,2, \ldots, D\}$ remain the random choice of indexes. Even though there is a random determining of $k$, it is important that it is different from $i$. The random number within the $[-1,1]$ range is $\varphi i, j$ controlling the generation of the source position of a neighbor food revolving around $x i, j$ while the modification stands for the visual comparison regarding the bee's neighbor food positions. In equation (4), there is a difference existing between the decrease in $x i, j$ and $x k, j$ parameters, as well as the position perturbation for the decrease in $x i, j$. Therefore, the more the search gets to the optimum solution within the search space; there is an adaptive reduction of the step length.

If there is a production of the parameter by this operation goes beyond the predetermined limit, an acceptable value would be set for the parameter. This study involves the parameter value going beyond the limit and being set to its limit value. There is a replacement of the abandoned food source by the bees by the scouts. There is also a simulation of the ABC algorithm through a random production of the position and replacement with uninhabited one. If the position in the $\mathrm{ABC}$ algorithm is not enhanced with the help of a predetermined amount of cycles known as limit, at this point the food source is considered abandoned. Once there is a production of each source position of the candidate $v i, j$, and an evaluation occurs by the artificial bee, there is a comparison of its performance to the one for $x i, j$. If the old source nectar is the same with or of lesser quality to the new food, a replacement can occur in the memory with the old one. If not, it is better to just leave the old one. What this means is that a covetous selection mechanism is adopted as the operation of selection between the current and also the previous food sources. There are four main selection processes adopted by $\mathrm{ABC}$ algorithm:

1. The artificial onlooker bees discover promising regions among those discovered so far by conducting a global selection process based on Equation 4.

2. The employed artificial bees in a certain region carry out a local selection process, based on the local information about that region available for those bees. (Inspired by the actual information used by the real bees, such as the color, fragrance and shape. However, even with real bees, the actual type of the nectar in the source is not identified until a bee reaches that source. These bees then use their memory to decide the optimal food source in the neighboring sources, using Equation 5.

3. Of the amount of nectar that is found in a candidate source is found to be better than another known to the bee, it selected the source with more nectar, according to the greedy selection process. The bee also forgets about past sources that have relatively limited amount of nectar and memorize the recognized new source. Otherwise, the currently being used source is kept in its memory.

4. Scouts carry out a random selection process to ensure balance between exploration and exploitation.

As these steps show, there are three parameters that are required in order to define the operation of the $\mathrm{ABC}$ algorithm, which are the food source count, the boundaries of the search space that surrounds the hive, as well as the maximum cycle number. The number of onlooker or employed bees is set according to the number of food sources that are surrounding the hive.

\subsection{The Sine-Cosine Algorithm}

The Sine Cosine Algorithm (SCA) also initiated a predefined number of random points in the D-dimensional space of the 
system being optimized. The next position $X$ of point $i$ at iteration $t$ out of $T$ total iterations is set by Equation (5) [17].

$$
X_{i}^{t+1}= \begin{cases}X_{i}^{t}+r_{1} \times \sin \left(r_{2}\right) \times\left|r_{3} P_{i}^{t}-X_{i}^{t}\right|, & r_{4}<0.5 \\ X_{i}^{t}+r_{1} \times \cos \left(r_{2}\right) \times\left|r_{3} P_{i}^{t}-X_{i}^{t}\right|, & r_{4} \geq 0.5\end{cases}
$$

where $P$ is the destination position, $r_{1}, r_{2}, r_{3}$ and $r_{4}$ are random numbers. $r_{4}$ is in the range [0,1], where its value determines which equation, the sine or cosine, is going to be used for computing the next position of the point. The parameter $r_{3}$ has a range of $[0,2]$ and brings a random weight for the destination in order to stochastically emphasize, when the value is greater than one, or deemphasize, when the value is less than 1, the effect of destination in defining the distance. The random number $r_{2}$ has a range $[-2,2]$ and defines the distance that the point is going to move, toward or outward the destination point. Finally, $r_{l}$ dictates the region of the next move, either between the current position and the destination point, or out of it. The effect of these number on the movement a certain point in the space is illustrated in Figure 4.

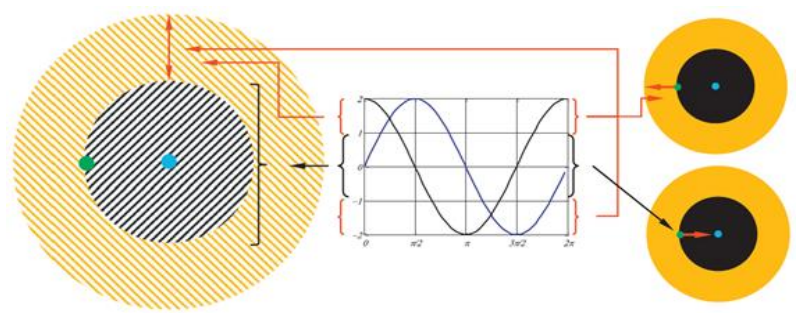

Fig 4: The random movement of a point in the SCA algorithm [17].

In order to balance the exploration and exploitation in the SCA algorithm, the value of $r_{I}$ is computed using Equation (6) [17].

$$
r_{1}=a-t \frac{a}{T}
$$

where $a$ is a predefined number that is multiplied by the ratio of the current iteration to the total number of iterations. The value of $r_{l}$ computed using this equation allows wider exploration at the beginning of the iteration, and more divergence toward the optimal coordinates as the iterations approach to the predefined maximum number of iterations.

\section{METHOD}

For each generator to be added to the grid, the optimizer is required to provide three values, the $x$ and $y$ coordinates of the generator, as well as its size. The boundaries of these values are set based on the geographical boundaries of the grid, e.g., distances from a reference point or longitude and latitude values, for the location, in addition to the maximum capacity of the generator for the size optimization. The busbar that is closest to the selected coordinates is selected by the proposed method in order to connect the DG unit to. The cost is calculated based on the total losses in the system and the voltage profile, when the DG units are connected to the selected busbars. The calculated cost in the proposed method provides a balanced weight to both the voltage profile and the total losses. However, a 5\% Pu voltage constraint is defined, so that, the cost is increased dramatically when such constraint is exceeded. Accordingly, the cost of the voltage profile is calculated as shown in Equation (7), which produces a value of zero when all voltages are equal to $1 \mathrm{Pu}$, and a maximum of 1 when all voltages are on the edge of the limits.
Otherwise, when the limits are exceeded, a cost of 100 is outputted to indicate the violation to the optimizer.

$$
\operatorname{Cost}_{V p}=\left\{\begin{array}{lr}
100 & \min \left\{b_{1}, b_{2}, \ldots, b_{n}\right\}<0.95 \\
100 & \max \left\{b_{1}, b_{2}, \ldots, b_{n}\right\}>1.05 \\
\frac{1}{n} \sum_{i=1}^{n}\left(b_{i}-1\right)^{2} / 0.0025 & \text { otherwise }
\end{array}\right.
$$

In addition to the voltage profile, the proposed method also includes the losses of the system in the cost computations. To balance the effect of these factors on the total cost, unless the voltages constraints are violated, the formula shown in Equation (8) is used to calculate the cost of losses, where $L$ is the loss in the grid before adding the new DG unit and $L_{D G}$ is the total loss in the grid after adding the DG unit to the selected busbar. According to this formula, a value of one is returned when the computed loss after adding the DG to the grid is equal to the total loss before adding the generator. However, if the added generator increases the total loss in the grid, a value greater than one is returned, whereas reducing the loss returns a value less than one. Finally, the total cost is delivered to the optimizer by adding the calculated costs for the loss and voltage profile.

$$
\operatorname{Cost}_{L}=\frac{L_{D G}}{L}
$$

The calculated costs are delivered to the optimizers per each particle for the set number of iterations, as shown in Algorithm 2. Then, based on the methodology of the optimizer, the optimal placement of the generator is selected. The performance of the proposed method is evaluated by measuring the average cost per each iteration of the three different optimizers, PSO, ABC and SCA. Each optimizer is run for ten times, ten iterations per each run with three particles. The average cost of the proposed method per each iteration is measured and compared to the average cost when the number of the busbar is used. The improvement in the

\begin{tabular}{|c|c|}
\hline \multicolumn{2}{|c|}{$\begin{array}{l}\text { Input: Search coordinates. } \\
\text { Output: Multi-objective cost value. }\end{array}$} \\
\hline Step1: & $x, y \leftarrow$ Read selected coordinates. \\
\hline Step2: & $\begin{array}{l}\left\{\mathrm{x}_{1}, \mathrm{x}_{2}, \ldots, \mathrm{x}_{\mathrm{n}}\right\},\left\{\mathrm{y}_{1}, \mathrm{y}_{2}, \ldots, \mathrm{y}_{\mathrm{n}}\right\} \leftarrow \text { Read } \\
\text { coordinates of the busbars. }\end{array}$ \\
\hline Step3: & $\begin{array}{l}\left\{\mathrm{d}_{1}, \mathrm{~d}_{2}, \ldots, \mathrm{d}_{\mathrm{n}}\right\} \leftarrow \text { Measure Euclidean distance } \\
\text { between } x, y \text { and each busbar. }\end{array}$ \\
\hline Step4: & Select busbar that has $\min \left\{\mathrm{d}_{1}, \mathrm{~d}_{2}, \ldots, \mathrm{d}_{\mathrm{n}}\right\}$. \\
\hline Step5: & $\begin{array}{l}\text { Simulate grid and measure } \operatorname{Cost}_{V P} \text { and } \operatorname{Cost}_{L} \\
\text { costs. }\end{array}$ \\
\hline Step6: & Return $\operatorname{Cost}_{V P}$ and $\operatorname{Cost}_{L}$ \\
\hline
\end{tabular}
cost, i.e., the reduction, illustrates the improvement in the performance of the optimizer.

Algorithm 2: Geolocation-aware DG placement optimization.

\section{PERFORMANCE EVALUATION}

Two models are used for the evaluation of the proposed method, which are the IEEE 69-bus distribution test system and the IEEE 33-bus radial distribution system. Per each system, two scenarios are simulated, in which one and two 
DG units are added with a maximum capacity of $1 \mathrm{MW}$. The parameters of the simulated systems are collected from [18] 69-bus distribution test system. Three optimization algorithms are used for the evaluation, which are the PSO, ABC and SCA. All experiments are implemented and conducted using Python programming language, where the PYPSA library [20] is used to simulate the power grids, whereas the Opytimizer library [21] is used to implement the optimizers. To illustrate the improvement in the performance of the optimizer when using the proposed geolocation-aware approach, the number of particles per each optimizer is set to five, where each optimizer is set to run for ten iterations. To avoid any biased evaluation, according to the random initialization of the particles, each optimizer is run for ten times and the average fitness per each iteration is used to illustrate the performance of the optimizer at that iteration. The performance of the proposed optimization approach when using the PSO optimizer, shown in Figure 5, illustrates the significant improvement in localizing and sizing the added generators. This improvement is illustrated by the lower cost value achieved by the same optimizer when using the proposed geolocation-aware approach, even in the case in which two generators are added to the 69-bus distribution system, where the standard approach has shown lower cost at the first iteration.Additionally, the performance of the $\mathrm{ABC}$ optimizer, shown in Figure 6,show the ability of the proposed

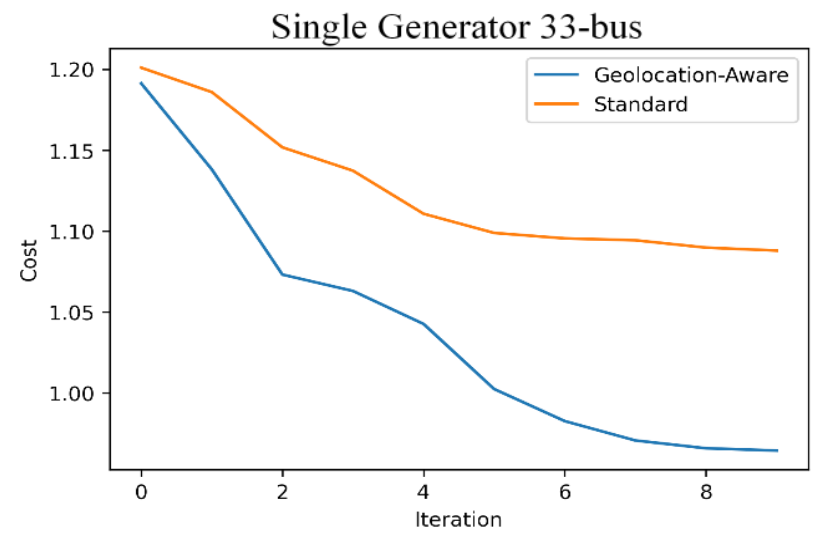

Single Generator 69-bus

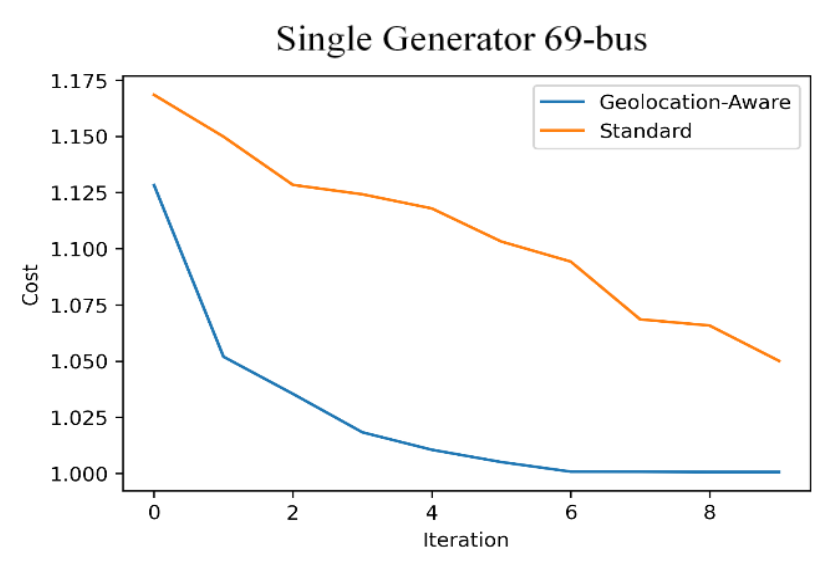

for the IEEE 33-bus radial distribution system and from [19] regarding the IEEE optimization approach to optimize the placement and sizing of the added generation unit in most of the scenarios, especially in more complex distribution system, i.e., the 69-bus system. The slightly better performance achieved by the standard placement approach when adding two generators to the 33-bus system is a result of lower number of busbars in the system, which has allowed the $\mathrm{ABC}$ optimizer to recognize the optimal placement faster, as the number of busbars is less in this model. Using the proposed approach, the optimizer needs to move the particles in relatively larger steps to change from one busbar to another, which is not the case when using the busbar number to specify the busbar. The proposed optimization approach has also been able to significantly improve the performance of the SCA optimizer for all scenarios, including when adding two generators to the 69-bus distribution system, as shown in Figure 7, in which the standard approach has achieved better performance for the first two iterations. However, the average cost by the end of the ten iterations is significantly lower than that in the standard approach, which illustrates the significance of the propose geolocation-aware optimization, as the changes that the optimizer is making comes along with the description of the grid.
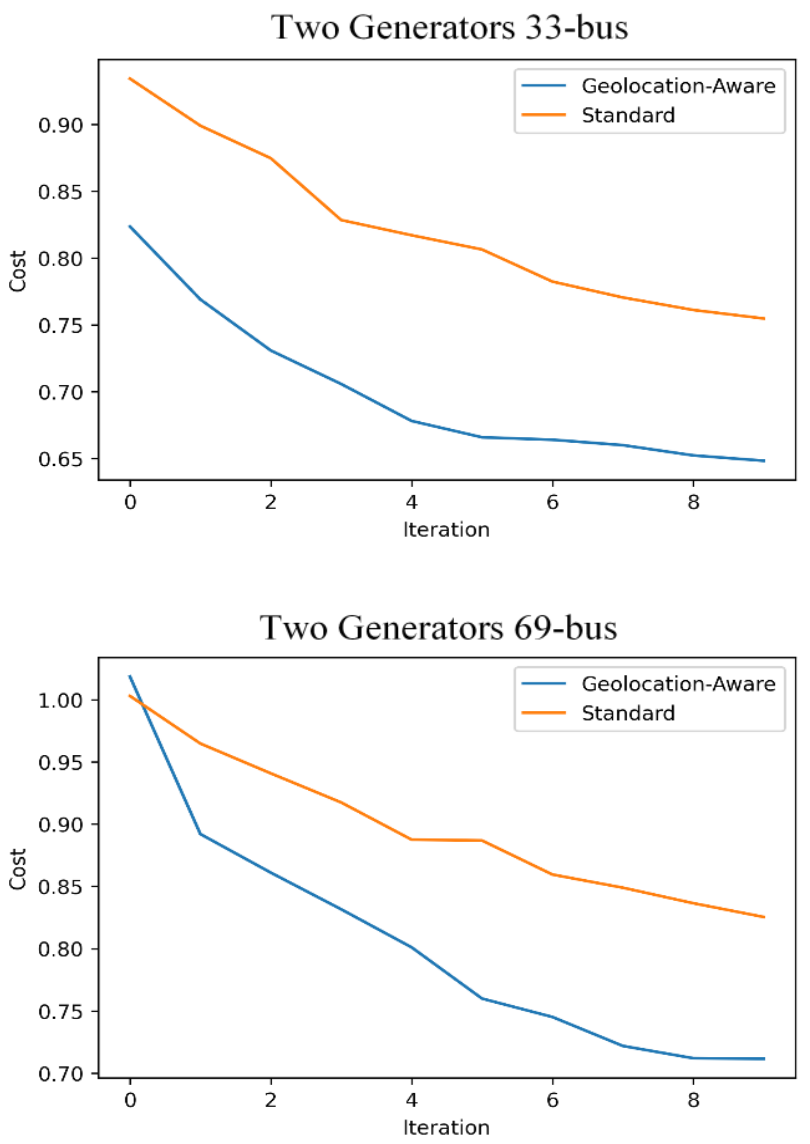

Fig 5: Performance of the PSO optimizer. Left: Adding a single generator; Right: Adding two generators; Top: 33-bus distribution system; Bottom: 69-bus distribution system 

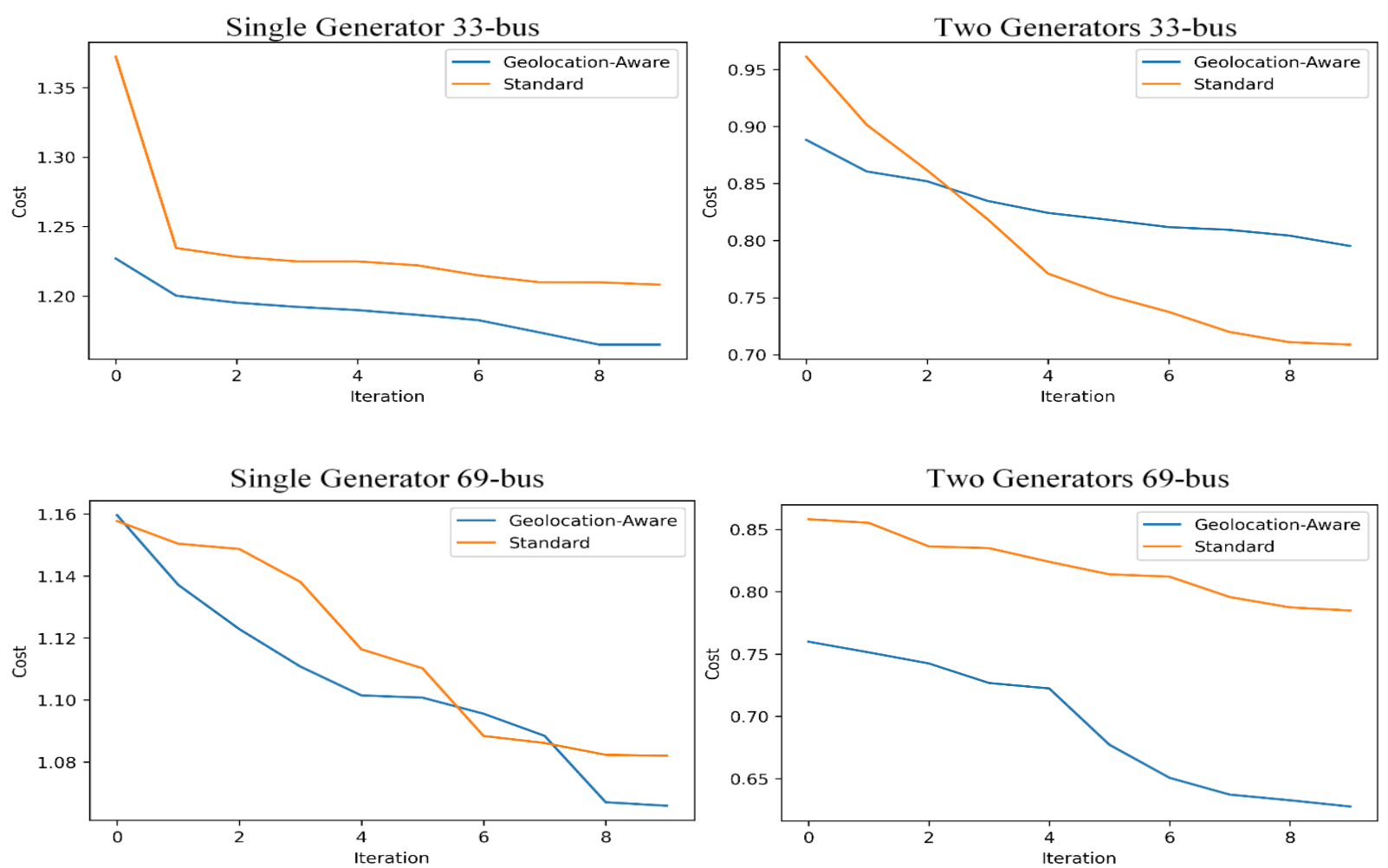

Fig 6:Performance of the ABC optimizer. Left: Adding a single generator; Right: Adding two generators; Top: 33-bus distribution system; Bottom: 69-bus distribution system
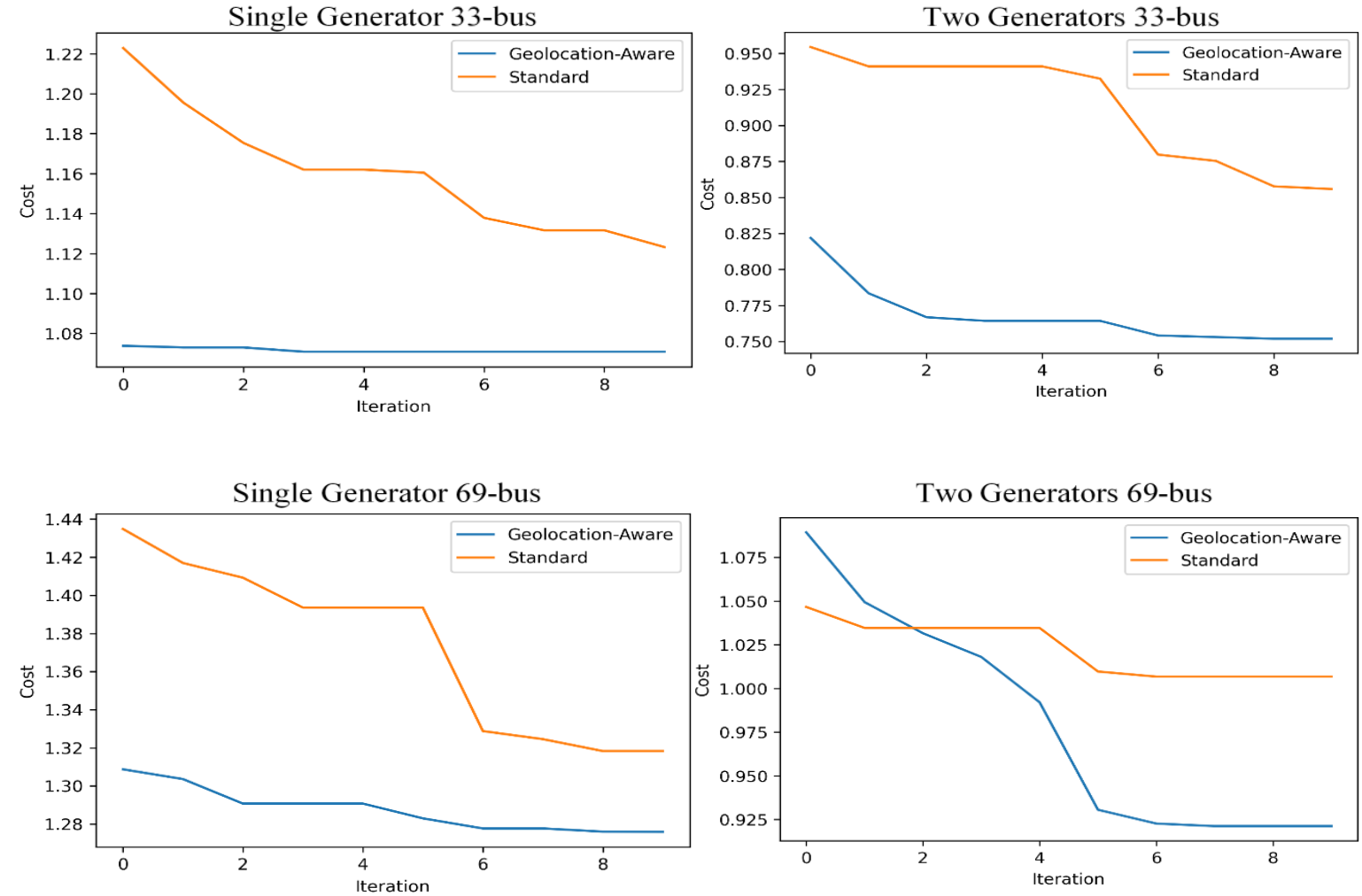

Fig 7: Performance of the SCA optimizer. Left: Adding a single generator; Right: Adding two generators; Top: 33-bus distribution system; Bottom: 69-bus distribution system

The average cost achieved by each of the classifiers in both scenarios, as well as averages achieved by each optimization approach, i.e., the standard and average approaches, are summarized in Table 1, which shows the significant improvement achieved by using the proposed geolocationaware approach. Overall, the proposed method has been able to reduce the cost, i.e., achieve better operation for the power grid, in all scenarios, accept the case in which the $\mathrm{ABC}$ 
optimizer is used to optimize the location and size of two DG units in the IEEE 69-bus radial distribution system. Additionally, the PSO has achieved the lowest average cost, in all scenarios, compared to the other optimizers, especially when the proposed optimization approach is used.
Accordingly, the use of the proposed optimization approach to find the optimal location and size of DG units to be added to existing power grids can significantly improve the operation conditions of that grid.

Table 1: Summary of the evaluation results

\begin{tabular}{|c|c|c|c|c|c|c|c|c|c|c|}
\hline \multirow[b]{3}{*}{ Optimizer } & \multicolumn{4}{|c|}{ IEEE 33-bus radial distribution system } & \multicolumn{4}{|c|}{ IEEE 69-bus radial distribution system } & & \\
\hline & \multicolumn{2}{|c|}{ Single DG Unit } & \multicolumn{2}{|c|}{ Two DG Units } & \multicolumn{2}{|c|}{ Single DG Unit } & \multicolumn{2}{|c|}{ Two DG Units } & \multicolumn{2}{|c|}{ Average } \\
\hline & Proposed & Standard & Proposed & Standard & Proposed & Standard & Proposed & Standard & Proposed & Standard \\
\hline PSO & 1.04 & 1.13 & 0.7 & 0.82 & 1.03 & 1.11 & 0.81 & 0.9 & 0.895 & 0.990 \\
\hline $\mathbf{A B C}$ & 1.1 & 1.12 & 0.69 & 0.82 & 1.19 & 1.24 & 0.83 & 0.79 & 0.953 & 0.993 \\
\hline SCA & 1.07 & 1.16 & 0.77 & 0.91 & 1.29 & 1.37 & 0.98 & 1.02 & 1.028 & 1.115 \\
\hline Average & 1.07 & 1.14 & 0.72 & 0.85 & 1.17 & 1.24 & 0.87 & 0.90 & 0.958 & 1.033 \\
\hline
\end{tabular}

In addition to the acceleration of the performance of the optimization algorithms, the improvement in the distributed grid, illustrated by the better voltage profile and less losses, are measured when using the PSO optimizer with the proposed optimization approach. This evaluation is conducted using the 33-bus radial distribution system, where one and two generators are added to the grid. As shown in Table 2, the proposed method has been able to significantly reduce the losses and improve the voltage profile, especially when two generators are added. When a single generator is added, the method proposed by Haider et al. [22] has been able to achieve similar results, according to the lower complexity of the search space. However, a significant improvement is achieved when using the propose optimization approach to add two generators, according to the better illustration of the search space to the PSO optimizer, which allows more efficient search of the optimal values.

Table 2: Characteristics of the 33-bus distribution system in different scenarios

\begin{tabular}{|c|c|c|c|c|c|}
\hline \multirow{2}{*}{ Method } & \multirow{2}{*}{$\begin{array}{c}\text { DG } \\
\text { Placement } \\
\text { and (size) }\end{array}$} & \multirow{2}{*}{$\begin{array}{c}\text { Losses } \\
\text { (KW) }\end{array}$} & \multicolumn{3}{|c|}{ Voltage (Pu) } \\
\hline & & & Min & Max & Average \\
\hline $\begin{array}{c}\text { Without } \\
\text { DG }\end{array}$ & - & 24.97 & 0.9880 & 1 & 0.9931 \\
\hline Standard & $16(1 \mathrm{MW})$ & 15.55 & 0.9925 & 1 & 0.9981 \\
\hline Proposed & $6(1 \mathrm{MW})$ & 14.04 & 0.9925 & 1 & 0.9979 \\
\hline $\begin{array}{l}\text { Haider et } \\
\text { al. [22] }\end{array}$ & $6(1 \mathrm{MW})$ & 14.04 & 0.9925 & 1 & 0.9979 \\
\hline Standard & $\begin{array}{l}14 \text { (1MW) } \\
30(1 \mathrm{MW})\end{array}$ & 7.32 & 0.9976 & 1 & 1 \\
\hline Proposed & $\begin{array}{l}14 \text { (1MW) } \\
31 \text { (1MW) }\end{array}$ & 6.7 & 0.9976 & 1 & 1 \\
\hline $\begin{array}{c}\text { Haider et } \\
\text { al. [22] }\end{array}$ & $\begin{array}{c}6(1 \mathrm{MW}) \\
16(1 \mathrm{MW})\end{array}$ & 11.36 & 0.9975 & 1 & 1 \\
\hline
\end{tabular}

\section{CONCLUSION}

With the growing significance of renewable energy, the addition of smaller distributed generation units has become the center of attention of several researchers. Specifying the size of the unit and the optimal placement have direct influence on the performance of the power grid, in terms of reliability and stability. Accordingly, a new optimization approach is proposed in this study, which provides the optimization algorithm with geolocation information to improve the efficiency of the search task of the optimization algorithm. The proposed approach has shown significant improvement in the performance of different types of classifiers, by finding better placements in shorter intervals. Additionally, the results show that the use of the proposed method has been able to further improve the power grid, by reducing the losses and improve the voltage profile, compared to the standard optimization and existing state-of-the-art methods. The propose method has been able to reduce the losses of the IEEE 33-bus radial distribution test system from $24.97 \mathrm{KW}$ to $6.7 \mathrm{KW}$ when two generators are added to the grid, with minimum voltage of $0.9976 \mathrm{Pu}$, whereas the maximum and average voltages across all busbars is $1 \mathrm{Pu}$. This improvement in the efficiency of the optimization allows recognizing the optimal placement and sizing for complex real-life scenarios, with a huge number of busbars, according to the efficient and more-suitable representation of the power grid to the optimization algorithm.

\section{REFERENCES}

[1] M. Andreasson, D. V. Dimarogonas, K. H. Johansson, and H. Sandberg, "Distributed vs. centralized power systems frequency control," in 2013 European Control Conference (ECC), 2013, pp. 3524-3529.

[2] J. Liu, W. Yao, J. Wen, J. Fang, L. Jiang, H. He, et al., "Impact of power grid strength and PLL parameters on stability of grid-connected DFIG wind farm," IEEE Transactions on Sustainable Energy, vol. 11, pp. 545557, 2019.

[3] V. Murty and A. Kumar, "Multi-objective energy management in microgrids with hybrid energy sources and battery energy storage systems," Protection and Control of Modern Power Systems, vol. 5, pp. 1-20, 2020.

[4] S. K. Injeti and V. K. Thunuguntla, "Optimal integration of DGs into radial distribution network in the presence of plug-in electric vehicles to minimize daily active power losses and to improve the voltage profile of the system using bio-inspired optimization algorithms," Protection 
and Control of Modern Power Systems, vol. 5, pp. 1-15, 2020.

[5] Y. Shen, W. Yao, J. Wen, H. He, and L. Jiang, "Resilient wide-area damping control using GrHDP to tolerate communication failures," IEEE Transactions on Smart Grid, vol. 10, pp. 2547-2557, 2018.

[6] K. Sun, W. Yao, J. Fang, X. Ai, J. Wen, and S. Cheng, "Impedance modeling and stability analysis of gridconnected DFIG-based wind farm with a VSC-HVDC," IEEE Journal of Emerging and Selected Topics in Power Electronics, vol. 8, pp. 1375-1390, 2019.

[7] X. Zhang, T. Tan, B. Zhou, T. Yu, B. Yang, and X. Huang, "Adaptive distributed auction-based algorithm for optimal mileage based AGC dispatch with high participation of renewable energy," International Journal of Electrical Power \& Energy Systems, vol. 124, p. 106371, 2021.

[8] B. Yang, L. Yu, Y. Chen, H. Ye, R. Shao, H. Shu, et al., "Modelling, applications, and evaluations of optimal sizing and placement of distributed generations: A critical state- of- the- art survey," International Journal of Energy Research, vol. 45, pp. 3615-3642, 2021.

[9] Z. Abdmouleh, A. Gastli, L. Ben-Brahim, M. Haouari, and N. A. Al-Emadi, "Review of optimization techniques applied for the integration of distributed generation from renewable energy sources," Renewable Energy, vol. 113, pp. 266-280, 2017.

[10] G. S. Chaurasia, A. K. Singh, S. Agrawal, and N. Sharma, "A meta-heuristic firefly algorithm based smart control strategy and analysis of a grid connected hybrid photovoltaic/wind distributed generation system," Solar Energy, vol. 150, pp. 265-274, 2017.

[11] I. Pisica, C. Bulac, and M. Eremia, "Optimal distributed generation location and sizing using genetic algorithms," in 2009 15th International Conference on Intelligent System Applications to Power Systems, 2009, pp. 1-6.

[12] K.-L. Du and M. Swamy, "Particle swarm optimization," in Search and Optimization by Metaheuristics, ed: Springer, 2016, pp. 153-173.
[13] J. L. Awange, B. Paláncz, R. H. Lewis, and L. Völgyesi, "Particle swarm optimization," in Mathematical Geosciences, ed: Springer, 2018, pp. 167-184.

[14] A. Engelbrecht, "Particle swarm optimization," in Proceedings of the Companion Publication of the 2014 Annual Conference on Genetic and Evolutionary Computation, 2014, pp. 381-406.

[15] S. Chavan and N. P. Adgokar, "An overview on particle swarm optimization: basic concepts and modified variants," International Journal of Science and Research, vol. 4, pp. 255-260, 2015.

[16] D. Karaboga, B. Basturk, A powerful and efficient algorithm for numerical function optimization: artificial bee colony (ABC) algorithm, Journal of Global Optimization 39 (3) (2007) 459-471.

[17] S. Mirjalili, " SCA: A Sine Cosine Algorithm for solving optimization," Knowledge-Based Systems, vol. 96, pp. 120-133, 2016.

[18] J. Ponoćko, Data Analytics-Based Demand Profiling and Advanced Demand Side Management for Flexible Operation of Sustainable Power Networks: Springer Nature, 2020

[19] J. Savier and D. Das, "Impact of network reconfiguration on loss allocation of radial distribution systems," IEEE Transactions on Power Delivery, vol. 22, pp. 2473-2480, 2007.

[20] T. Brown, J. Hörsch, and D. Schlachtberger, "PyPSA: Python for power system analysis," arXiv preprint arXiv:1707.09913, 2017.

[21] G. H. de Rosa, D. Rodrigues, and J. P. Papa,"Opytimizer: A nature-inspired python optimizer," arXiv preprint arXiv:1912.13002, 2019.

[22] W. Haider, S. Hassan, A. Mehdi, A. Hussain, G. O. M. Adjayeng, and C.-H. Kim, "Voltage profile enhancement and loss minimization using optimal placement and sizing of distributed generation in reconfigured network," Machines, vol. 9, p. 20, 2021. 